\title{
Origin of radiative recombination and manifestations of localization effects in GaAs/GaNAs core/shell nanowires
}

Shula Chen, Stanislav Filippov, Fumitaro Ishikawa, Weimin Chen and Irina Buyanova

\author{
Linköping University Post Print
}

Tweet

N.B.: When citing this work, cite the original article.

Original Publication:

Shula Chen, Stanislav Filippov, Fumitaro Ishikawa, Weimin Chen and Irina Buyanova, Origin of radiative recombination and manifestations of localization effects in GaAs/GaNAs core/shell nanowires, 2014, Applied Physics Letters, (105), 25, 253106.

http://dx.doi.org/10.1063/1.4905090

Copyright: American Institute of Physics (AIP) http://www.aip.org/

Postprint available at: Linköping University Electronic Press

http://urn.kb.se/resolve?urn=urn:nbn:se:liu:diva-113778 


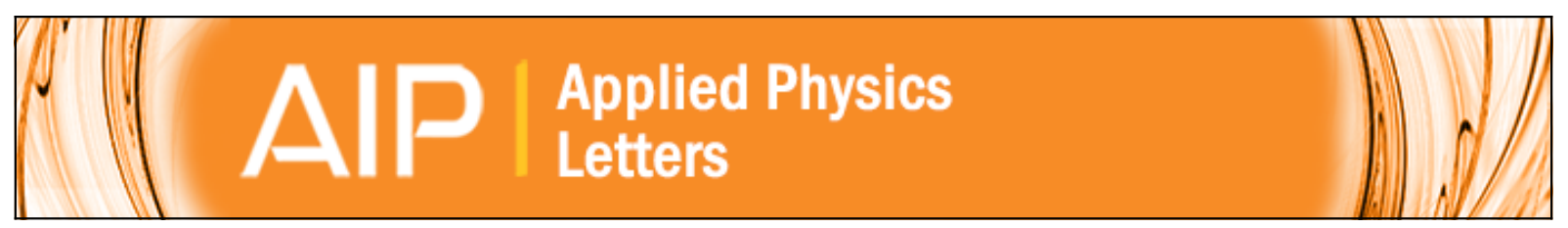

\section{Origin of radiative recombination and manifestations of localization effects in GaAs/GaNAs core/shell nanowires}

S. L. Chen, S. Filippov, Fumitaro Ishikawa, W. M. Chen, and I. A. Buyanova

Citation: Applied Physics Letters 105, 253106 (2014); doi: 10.1063/1.4905090

View online: http://dx.doi.org/10.1063/1.4905090

View Table of Contents: http://scitation.aip.org/content/aip/journal/apl/105/25?ver=pdfcov

Published by the AIP Publishing

\section{Articles you may be interested in}

Mechanism for radiative recombination and defect properties of GaP/GaNP core/shell nanowires

Appl. Phys. Lett. 101, 163106 (2012); 10.1063/1.4760273

Long minority carrier lifetime in Au-catalyzed GaAs/AlxGa1-xAs core-shell nanowires

Appl. Phys. Lett. 101, 023111 (2012); 10.1063/1.4735002

Carrier localization and nonradiative recombination in yellow emitting InGaN quantum wells Appl. Phys. Lett. 96, 031906 (2010); 10.1063/1.3293298

Measurement of the Auger recombination rate in p-type $0.54 \mathrm{eV}$ GalnAsSb by time-resolved photoluminescence Appl. Phys. Lett. 83, 3317 (2003); 10.1063/1.1621455

On the nature of radiative recombination in GaAsN

Appl. Phys. Lett. 81, 4368 (2002); 10.1063/1.1526913

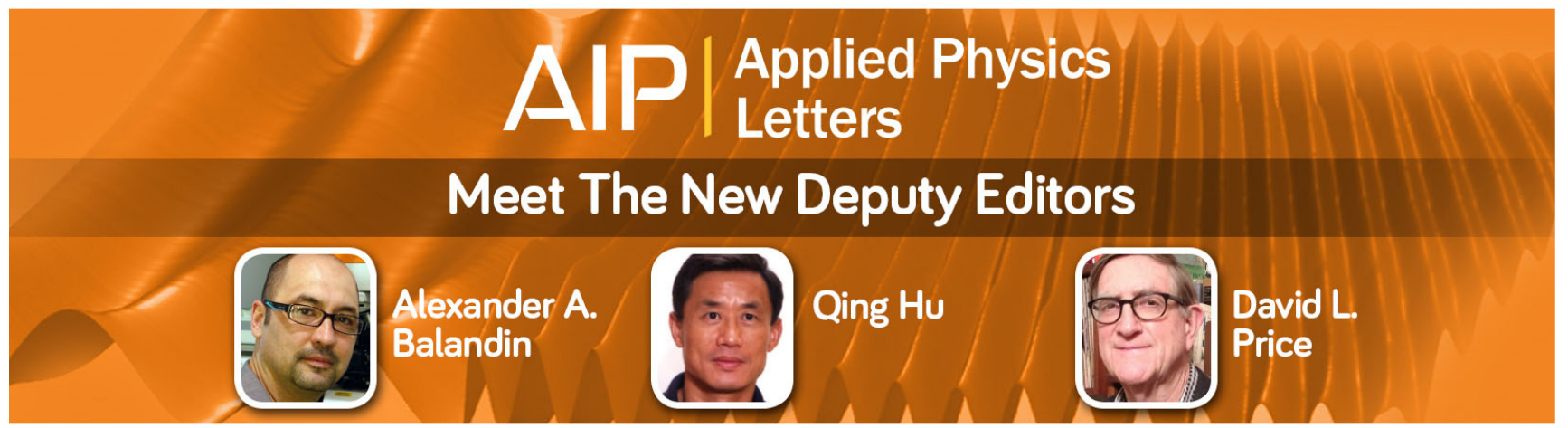




\title{
Origin of radiative recombination and manifestations of localization effects in GaAs/GaNAs core/shell nanowires
}

\author{
S. L. Chen, ${ }^{1}$ S. Filippov, ${ }^{1}$ Fumitaro Ishikawa,${ }^{2}$ W. M. Chen,${ }^{1}$ and I. A. Buyanova ${ }^{1, a)}$ \\ ${ }^{1}$ Department of Physics, Chemistry and Biology, Linköping University, 58183 Linköping, Sweden \\ ${ }^{2}$ Graduate School of Science and Engineering, Ehime University, 790-8577 Matsuyama, Japan
}

(Received 21 November 2014; accepted 15 December 2014; published online 24 December 2014)

\begin{abstract}
Radiative carrier recombination processes in GaAs/GaNAs core/shell nanowires grown by molecular beam epitaxy on a $\mathrm{Si}$ substrate are systematically investigated by employing microphotoluminescence ( $\mu$-PL) and $\mu$-PL excitation ( $\mu$-PLE) measurements complemented by time-resolved PL spectroscopy. At low temperatures, alloy disorder is found to cause localization of photo-excited carriers leading to predominance of optical transitions from localized excitons (LE). Some of the local fluctuations in $\mathrm{N}$ composition are suggested to lead to strongly localized three-dimensional confining potential equivalent to that for quantum dots, based on the observation of sharp and discrete PL lines within the LE contour. The localization effects are found to have minor influence on PL spectra at room temperature due to thermal activation of the localized excitons to extended states. Under these conditions, photo-excited carrier lifetime is found to be governed by non-radiative recombination via surface states which is somewhat suppressed upon $\mathrm{N}$ incorporation. (c) 2014 AIP Publishing LLC. [http://dx.doi.org/10.1063/1.4905090]
\end{abstract}

III-V semiconductor nanowires (NWs), which provide both an optical active medium and well-shaped cavity, have been drawing enormous research interest as potential building blocks for a variety of optoelectronic devices including solar cells, photo-detectors, and light-emitting devices. ${ }^{1,2}$ Thanks to advances in growth techniques, NWs can now be fabricated not only from binary compounds but also ternary alloys with desired alloy compositions and also with a preferred design (e.g., radial core/shell or axial heterostructures), which allows tailoring and engineering of material properties at the nanoscale. Additionally, the NW growth can be performed on foreign substrates which render the possibility of integration of the NW-based optoelectronic devices with the mature microelectronic technology based on Si. Up to date, optical output from NWs has been demonstrated over a wide spectral range from ultraviolet to near-infrared. ${ }^{3-10}$ Intense research efforts are currently being undertaken to push the emission towards longer wavelengths of great significance in photovoltaics, medical diagnosis, and for fibre-optic data/telecommunication.

Among the most promising materials for the aforementioned long-wavelength applications are $\mathrm{Ga}(\mathrm{In}) \mathrm{NAs}$ alloys, which belong to the so-called dilute nitride semiconductors. The substitution of a small amount of As atoms in $\mathrm{Ga}$ (In)As by $\mathrm{N}$ atoms substantially modifies the band structure, giving rise to a reduced bandgap energy due to the bandgap bowing effect. $^{11,12}$ Moreover, strong spin-dependent recombination in these materials mediated by defects allows achieving a record-high electron spin polarization of up to $43 \%$ at room temperature (RT), which makes the $\mathrm{Ga}(\mathrm{In}) \mathrm{NAs}$ alloys an ideal materials system for innovative spin filters, spin detectors, amplifiers, and nuclear spin hyperpolarizers operational at RT. ${ }^{13-16}$ Most recently, it was shown that GaNAs can be

\footnotetext{
a) Author to whom correspondence should be addressed. Electronic mail: iribu@ifm.liu.se
}

grown not only in planar but also in NW architecture, i.e., as a shell layer in GaAs/GaNAs core/shell structures. ${ }^{17,18}$ This raises the prospect of combining advantages of this materials system with those offered by the one-dimensional (1D) NW architecture.

In the case of bulk $\mathrm{Ga}(\mathrm{In}) \mathrm{NAs}$, alloying with $\mathrm{N}$ also affects material quality. For example, Ga(In)NAs alloys exhibit very strong fluctuations in the conduction band (CB) edge due to alloy disorder since even a small change in the $\mathrm{N}$ content $[\mathrm{N}]$ leads to a significant change of the bandgap energy due to the large bandgap bowing. ${ }^{11,19}$ This influences both transport and optical properties of the alloys and affects performance of the related photonic devices. $\mathrm{N}$ incorporation can also promote formation of various defects that act as efficient recombination centers degrading internal quantum efficiency of the dilute nitrides. ${ }^{20-22}$ Though these effects are well understood for planar GaNAs-based structures, their role in 1D NWs, which are grown under different growth conditions as compared with the planar growth, remains unknown. In this work, we clarify this important issue based on systematic optical studies employing macro- and micro $(\mu)$ photoluminescence (PL) complemented by $\mu$-PL excitation ( $\mu$-PLE) and time-resolved PL measurements.

The studied zinc-blende (ZB) GaAs/GaNAs core/shell NWs were grown ${ }^{16,17}$ on a (111) Si substrate by plasmaassisted molecular beam epitaxy using Ga as a catalyst, with [N] of $0.1 \%$ and $0.5 \%$. For comparison, reference GaAs NWs without the GaNAs shells were grown under identical conditions as the GaAs cores in the core/shell structures. A representative scanning electron microscopy image of the GaAs/GaN ${ }_{0.005} \mathrm{As}_{0.995} \mathrm{NWs}$ is shown in Fig. 1(a). The NWs are rather uniform in sizes with an average length of $\sim 4.5 \mu \mathrm{m}$, a diameter of 400(200) $\mathrm{nm}$ for the GaAs/GaNAs core/shell (GaAs) NWs, and a well-defined hexagonal crosssection (see the inset in Fig. 1(a)). For $\mu$-PL studies, the nanowires were mechanically transferred onto another $\mathrm{Si}$ 


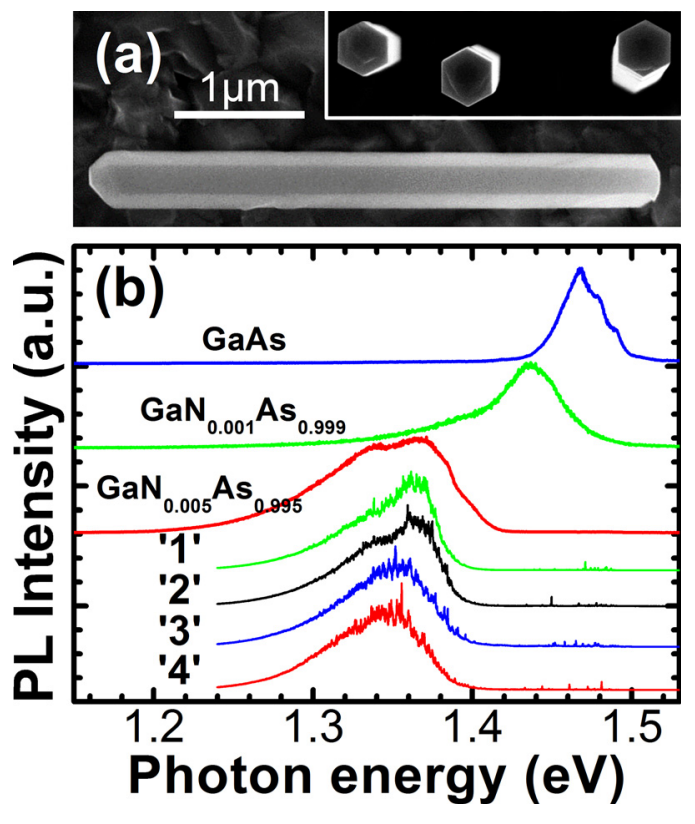

FIG. 1. (a) A schematic SEM image of the studied GaAs/GaNAs core/shell NWs with $[\mathrm{N}]=0.5 \%$. (b) Representative PL spectra of the investigated structures obtained at $5 \mathrm{~K}$. The three top spectra were measured from ensembles of GaAs NWs, GaAs/ $\mathrm{GaN}_{0.001} \mathrm{As}_{0.999}$, and $\mathrm{GaAs} / \mathrm{GaN}_{0.005} \mathrm{As}_{0.995}$ core/ shell NWs. The four lower spectra labeled as "1"-"4" are results from the $\mu$-PL measurements performed on four single $\mathrm{GaAs} / \mathrm{GaN}_{0.005} \mathrm{As}_{0.995}$ core/ shell NWs.

substrate. The measurements were performed in a backscattering geometry using a $50 \times$ objective lens. A $440 \mathrm{~nm}$ solid-state diode laser and a tunable Ti:Sapphire laser were used as excitation sources. The latter was also employed for $\mu$-PLE measurements. The PL signal was detected using a CCD camera assembled with a grating monochromator. Time-resolved PL measurements were carried out at RT by using as an excitation source a Ti: Sapphire picosecond pulsed laser emitting at $740 \mathrm{~nm}$, with a pulse repetition rate of $76 \mathrm{MHz}$. The transient PL signal was recorded by a streak camera system.

Typical $5 \mathrm{~K}$ PL spectra measured from NW ensembles are shown by three upper curves in Fig. 1(b). In the case of the GaAs NWs, the PL emission peaks at $\sim 1.467 \mathrm{eV}$ and contains several weaker features at higher energies. Such emission spectrum is typical for GaAs NWs with mixed ZB and wurtzite WZ crystal structure and has been shown to arise from excitonic transitions at the interfaces between the WZ and ZB phases and at staking faults. ${ }^{23-26}$ Formation of the GaNAs shell has several effects on the PL spectra. First, the core emission is substantially quenched, likely due to its absorption by the outer GaNAs shell with a lower bandgap and excitation transfer from the core to the shell layer. Second, the PL peak position shifts to lower energies with increasing $[\mathrm{N}]$. This indicates that the PL emission in the core/shell NWs originates from the GaNAs shell with a smaller bandgap. Additionally, the PL band becomes broad and asymmetric, with a long tail at low energies. Such PL lineshape is typical for GaNAs alloys ${ }^{19,27,28}$ and is characteristic for radiative transitions of localized excitons (LE) trapped within band tail states caused by fluctuations in $[\mathrm{N}]$.

To examine the distribution of localized states in individual NW, we performed $\mu$-PL measurements on single NWs. Representative PL spectra from four NWs are shown in the lower part of Fig. 1(b) and are labeled as "1"-"4." One notices that (i) the PL spectra from individual NWs are narrower than that measured from the NW ensemble; and (ii) the PL peak position changes from wire to wire within $\sim 20 \mathrm{meV}$. This point towards variations in $[\mathrm{N}]$ between the wires, as will be further confirmed from temperaturedependent $\mu$-PL and $\mu$-PLE measurements described below. Moreover, one can see that the broad emission band contains a number of rather sharp lines. These PL lines can be attributed to recombination of excitons localized within certain spatial regions of the NWs by compositional disorder. Indeed, local fluctuations in $[\mathrm{N}]$ may create a threedimensional confinement acting as quantum dots $(\mathrm{QD})^{29}$ that are distributed along the NWs. Additionally, the sharp lines can also stem from excitons trapped at stacking faults caused by WZ/ZB polymorphism. We, however, believe that for the studied GaNAs NWs the first localization mechanism dominates. This is because the sharp PL lines within the broad LE band can also be detected in $\mu$-PL spectra from the reference GaNAs epilayer, though with a much higher density. They can be resolved in the studied individual NWs due to a small excitation volume determined by the excitation spot and the NW thickness. We will therefore refer to these sharp lines as the QD emissions.

The suggested origin of the PL emission as being due to the LE recombination is further supported by temperature dependent $\mu$-PL measurements. The results of these measurements are depicted in Fig. 2(a) taking as example results

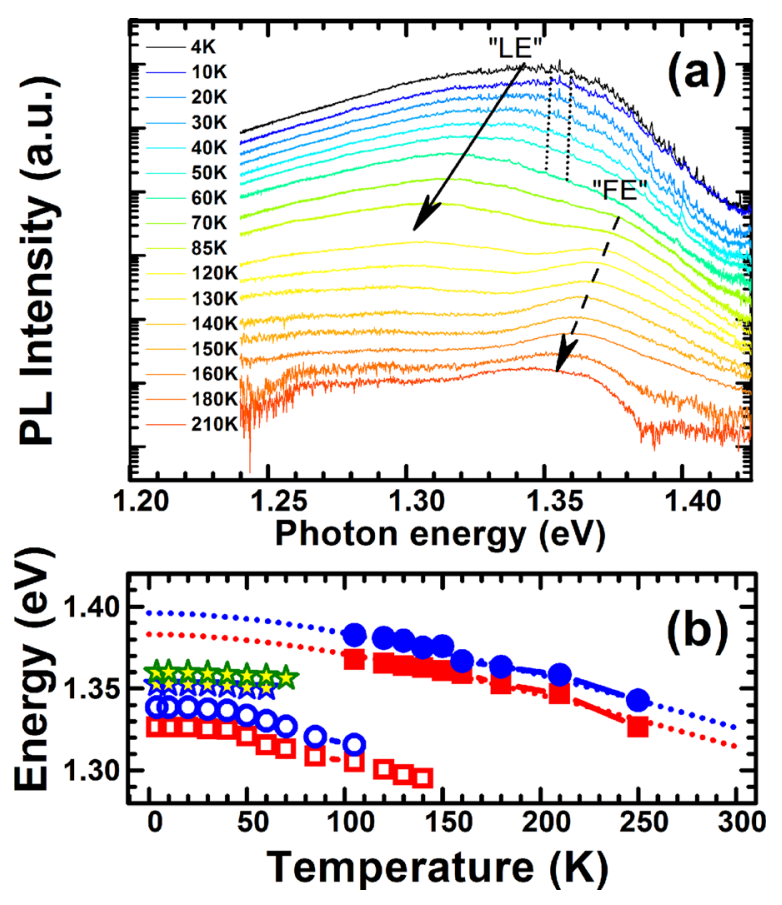

FIG. 2. (a) Temperature dependent PL spectra measured from the single NW "4." The solid and dashed arrows mark the peak positions of the LE and FE PL components, respectively. The dotted lines illustrate T-dependence of spectral positions of several sharp PL lines within the LE contour. (b) The peak position of the LE (the open symbols) and FE (the solid symbols) PL bands measured as a function of temperature from the NW " 2 " (the circles) and NW "4" (the squares). The dotted lines are expected T-dependences of the GaNAs bandgap energy based on the BAC model for the alloys with $[\mathrm{N}]=0.5 \%$ (the upper curve) and $0.56 \%$ (the lower curve). The stars denote the energies of the sharp PL lines as indicated by the dotted lines in (a). 
from the NW "4." With increasing temperature (T), the peak position of the overall PL band (labelled as "LE" in Fig. 2(a)) undergoes a red shift, as marked by the solid arrow. Above $85 \mathrm{~K}$, an additional PL band appears at the high energy side of the PL spectra (labelled as "FE" in Fig. 2(a)) and gains its intensity with rising T. The peak position of the FE band also shifts to lower energies with increasing $\mathrm{T}$ (shown by the dashed arrow in Fig. 2(a)), but with a slower rate as compared with the LE emission. As a result, temperature dependences of the PL maximum position exhibit an apparent "S-shape" dependence, as can also be seen from Fig. 2(b), where peak positions of the LE and FE transitions for the NWs "2" (dots) and "4" (squares) are shown. This "S-shape" T-dependence is typical for LE transitions and is common in dilute nitrides. ${ }^{19,27,28}$ Thermally activated escape of excitons from the localized states, which starts from the shallowest localization potential, will cause a strong red shift of the LE band with increasing $\mathrm{T}$. We note, however, that this strong shift is not expected for individual PL lines that contribute to the LE emission as each of them represents the radiative transition within a particular localization potential leading to the exciton confinement. The spectral positions of each QD emission should then reflect a $\mathrm{T}$-induced shift of the bandgap energy of the specific region, which is rather weak for $\mathrm{T}<80 \mathrm{~K}$. This is indeed observed experimentally-see Fig. 2(a), where the spectral positions of several QD emissions are indicated by the dotted lines. They are also shown by the stars in Fig. 2(b). At higher T, complete thermal depopulation of the localized states will lead to the activation of radiative recombination from free excitons/carriers giving rise to the FE PL component. The weaker T-induced shift of this emission will follow the T-induced reduction of the GaNAs bandgap energy $\mathrm{E}_{\mathrm{g}}^{\mathrm{GaNAs}}$.

According to previous studies of planar dilute nitrides, ${ }^{30,31}$ the thermal variation of the bandgap energy in these materials can be accurately modeled within the framework of the so-called band-anticrossing-model (BAC) ${ }^{11,12}$ that predicts a slow-down of the $\mathrm{E}_{\mathrm{g}}(\mathrm{T})$ dependence with increasing $[\mathrm{N}]$ so that

$$
\begin{aligned}
E_{g}^{G a N A s}(T)= & \frac{1}{2}\left[\left(E_{g}^{G a A s}(T)+E_{N}\right)\right. \\
& \left.-\sqrt{\left(E_{g}^{G a A s}(T)-E_{N}\right)^{2}+4 x C_{N M}^{2}}\right] .
\end{aligned}
$$

Here, $E_{g}{ }^{G a A s}(T)$ is the T-dependent bandgap of GaAs, $E_{N}=1.65 \mathrm{eV}$ is the energy of the localized $\mathrm{N}$ level relative to the valence band edge that is assumed to be T-independent, and $\mathrm{x}$ is the $\mathrm{N}$ fraction. $C_{N M}$ is the coupling parameter that is equal to $2.5 \mathrm{eV}$ for GaAs. ${ }^{30}$ By fitting measured T-dependences of the FE PL maximum position using Eq. (1), we can estimate $\mathrm{N}$ compositions in the individual NWs. The corresponding fitting results are shown by the dotted lines in Fig. 2(b) and yield $[\mathrm{N}]=0.50 \%(0.56 \%)$ for the NW " 2 " ("4"). We note that the obtained values are close to the highest and the lowest $[\mathrm{N}]$ in the studied NWs, which shows that the variation in the $\mathrm{N}$ content between the wires is rather small, i.e., within $0.07 \%$.
Further information about overall homogeneity of the GaNAs alloy within the individual NW can be obtained from $\mu$-PLE measurements. Shown in Figure 3 are $\mu$-PLE spectra of the NW " 2 " detected at $\mathrm{E}_{\mathrm{det}}=1.355 \mathrm{eV}$ (the solid line) and $1.302 \mathrm{eV}$ (the dashed line), respectively. For easy reference, we also show the PL spectrum (the dotted line) of this NW where $E_{\text {det }}$ used for the PLE measurements are indicated by the vertical lines. One notices that the PL intensity is practically constant for excitation energies $\mathrm{E}_{\mathrm{exc}}$ above $1.42 \mathrm{eV}$ but rapidly decreases for lower $\mathrm{E}_{\mathrm{exc}}$. Moreover, the cut-off of the measured PLE spectra depends on the detection energy, and is shifted to lower energies with decreasing $\mathrm{E}_{\mathrm{det}}$. Since PLE spectra are mainly determined by optical absorption transition between extended states, these changes in the PLE cut-off energy should be related to variations of the $\mathrm{N}$ composition within the $\mathrm{NW}$ either in radial or axial directions. By linearly extrapolating the dependence of the squared PL intensity $\left(\mathrm{I}_{\mathrm{PL}}\right)$ as a function of $\mathrm{E}_{\text {exc }}$, see the inset in Fig. 3, the local $\mathrm{E}_{\mathrm{g}}{ }^{\mathrm{GaNAs}}$ within the NW can be estimated to vary between $1.388 \mathrm{eV}$ and $1.394 \mathrm{eV}$. From Eq. (1), this corresponds to the variation of [N] within $0.04 \%$. This indicates that the broadening of the GaNAs-related PL is caused not only by alloy disorder responsible for the formation of band tail states but also by long-range variations in [N] affecting energies of the extended states. The long-range variations in $[\mathrm{N}]$ also lead to the appearance of two PL components within the LE emission that can be seen in the PL spectra of some of the wires (e.g., wire "2") and become broader in the PL spectra of the NW ensemble, as a result of statistical averaging between the wires-see Fig. 1(b). From Fig. 1(b), this effect becomes less severe for the GaAs/ $\mathrm{GaN}_{0.001} \mathrm{As}_{0.999} \mathrm{NWs}$, as a reduced $\mathrm{N}$ composition is also expected to lead to a reduced magnitude in the long-range composition variations. We note that this effect is not specific for the NW growth but is equally present in planar GaNAs.

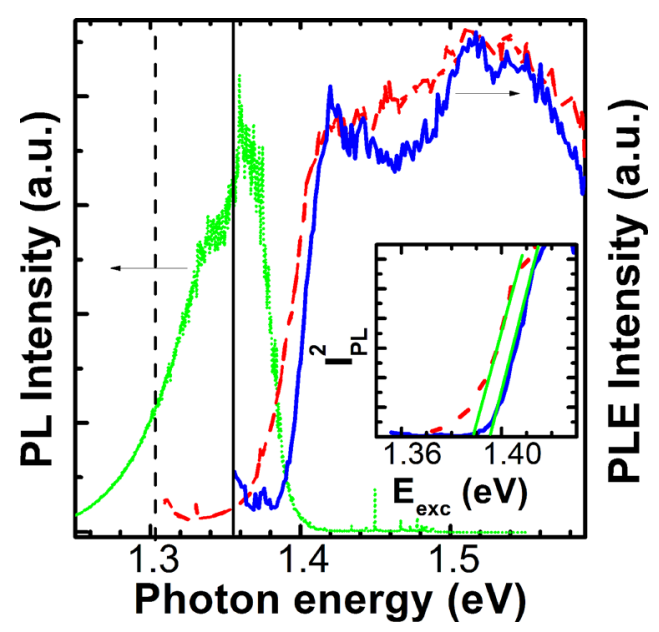

FIG. 3. $\mu$-PLE spectra measured from the NW " 2 " at the detection energies of $1.352 \mathrm{eV}$ (the solid line, blue) and $1.313 \mathrm{eV}$ (the dashed line, red), respectively. The inset shows the dependence of the squared PL intensity $\left(\mathrm{I}_{\mathrm{PL}}\right)$ as a function of the excitation energy $E_{\text {exc }}$ used to extrapolate the GaNAs bandgap energy (solid green lines). The PL spectrum of this NW is shown by the dotted line (green), where the detection energies used for the PLE measurements are indicated by the vertical lines. 
From Fig. 2(a), it is also obvious that a $\mathrm{T}$ increase causes an overall quenching of the PL emission due to activation of competing non-radiative recombination (NRR) that limits photo-excited carrier lifetime and degrades internal quantum efficiency of the NWs. This NRR can be either surface-related or caused by bulk defects acting as NRR centers. The former is known to be enhanced in nanostructures due to a large surface-to-volume ratio and is particularly severe in the GaAs NWs where surface passivation by either an outer shell ${ }^{32-34}$ or treatments with sulfide-based chemicals ${ }^{35,36}$ were utilized to improve their internal quantum efficiency. On the other hand, dilute nitrides are known to suffer from NRR via defects in the bulk promoted by the presence of N. ${ }^{20-22,37}$ Importance of these processes for the studied NWs was evaluated from timeresolved PL measurements. Representative RT time decays of the integrated PL intensity from the studied NWs are shown in Fig. 4. For comparison, also shown is the PL decay from the reference $\mathrm{GaN}_{0.005} \mathrm{As}_{0.995}$ epilayer. For all the structures, the PL decay is nearly single exponential with a decay time ranging from 4 ps in GaAs NWs to 8 ps in the GaAs/ $\mathrm{GaN}_{0.005} \mathrm{As}_{0.995} \mathrm{NWs}$ and to $12 \mathrm{ps}$ in the $\mathrm{GaN}_{0.005} \mathrm{As}_{0.995}$ epilayer. The obtained decay times are governed by NRR processes as they are substantially shorter than the radiative lifetime in GaAs, i.e., around $6 \mathrm{~ns}$ at $5 \mathrm{~K}$ in bulk GaAs (Ref. 38) and is even longer at RT. The observed shorter lifetime in the GaNAs NWs as compared with that in the planar GaNAs epilayer with the same $[N]$ can be attributed to the contribution of the surface recombination. Most unexpectedly, the surface recombination seems to become less severe upon $\mathrm{N}$ incorporation, as the PL decay in NWs slows down with increasing $[\mathrm{N}]$-see Fig. 4. This effect is opposite to that observed in GaNP-based NWs. ${ }^{39}$ Though the exact mechanism for the observed slow down is still unknown, a possible cause could be a N-induced modification of the surface states that are responsible for NRR at/near the surfaces of the studied GaNAs NWs. Such a reduction in surface recombination could, therefore, be beneficial for future applications of the GaNAs alloys in nano-scale light emitters.

In summary, we have investigated effects of alloying with nitrogen on the optical properties of the GaAs/GaNAs

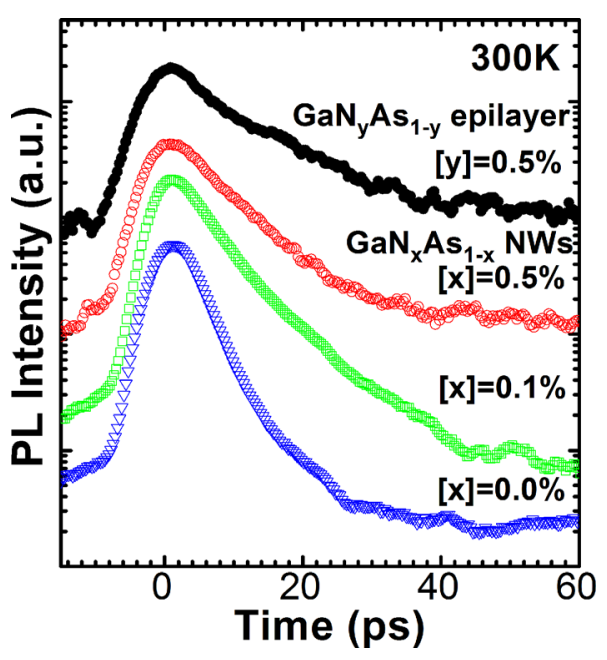

FIG. 4. Room temperature PL transients measured from ensembles of GaAs NWs, and GaAs/GaNAs core/shell NWs. The PL decay of the reference GaNAs epilayer with $[\mathrm{N}]=0.5 \%$ is also shown. core-shell NWs. We have shown that the low temperature PL emission in these structures is dominated by recombination of excitons trapped by alloy disorder. Some of these local regions are suggested to act as quantum dots, based on the observation of sharp and discrete PL lines within the LE contour in $\mu$-PL spectra from individual NWs. Good overall uniformity of the $\mathrm{N}$ content between different NWs and also within a single NW is also concluded based on temperaturedependent $\mu$-PL and $\mu$-PLE measurements performed on individual NWs. Localization effects are found to have minor influence on the RT PL spectra due to thermal activation of the localized excitons to extended states. Under these conditions, carrier lifetime is found to be governed by nonradiative recombination via surface states, which interestingly is somewhat suppressed upon $\mathrm{N}$ incorporation.

${ }^{1}$ R. X. Yan, D. Gargas, and P. D. Yang, Nat. Photonics 3, 569 (2009).

${ }^{2}$ Y. Li, F. Qian, J. Xiang, and C. M. Lieber, Mater. Today 9, 18 (2006).

${ }^{3}$ M. A. Zimmer, J. Bao, F. Capasso, S. Muller, and C. Ronning, Appl. Phys. Lett. 93, 051101 (2008).

${ }^{4}$ S. Chu, G. P. Wang, W. H. Zhou, Y. Q. Lin, L. Chernyak, J. Z. Zhao, J. Y. Kong, L. Li, J. J. Ren, and J. L. Liu, Nat. Nanotechnol. 6, 506 (2011).

${ }^{5}$ S. Gradecak, F. Qian, Y. Li, H. G. Park, and C. M. Lieber, Appl. Phys. Lett. 87, 173111 (2005).

${ }^{6}$ X. Duan, Y. Huang, R. Agarwal, and C. M. Lieber, Nature 421, 241 (2003).

${ }^{7}$ R. Agarwal, C. J. Barrelet, and C. M. Lieber, Nano Lett. 5, 917 (2005).

${ }^{8}$ F. Qian, Y. Li, S. Gradecak, H. G. Park, Y. J. Dong, Y. Ding, Z. L. Wang, and C. M. Lieber, Nat. Mater. 7, 701 (2008).

${ }^{9}$ Y. Xiao, C. Meng, P. Wang, Y. Ye, H. K. Yu, S. S. Wang, F. Gu, L. Dai, and L. M. Tong, Nano Lett. 11, 1122 (2011).

${ }^{10}$ D. Saxena, S. Mokkapati, P. Parkinson, N. Jiang, Q. Gao, H. H. Tan, and C. Jagadish, Nat. Photonics 7, 963 (2013).

${ }^{11}$ For a review, see, e.g., Physics and Applications of Dilute Nitrides, edited by I. A. Buyanova and W. M. Chen (Taylor \& Francis, London, 2004).

${ }^{12}$ W. Shan, W. Walukiewicz, J. W. Ager III, E. E. Haller, J. F. Geisz, D. J. Friedman, J. M. Olson, and S. R. Kurtz, Phys. Rev. Lett. 82, 1221 (1999).

${ }^{13}$ X. J. Wang, I. A. Buyanova, F. Zhao, D. Lagarde, A. Balocchi, X. Marie, C. W. Tu, J. C. Harmand, and W. M. Chen, Nat. Mater. 8, 198 (2009).

${ }^{14}$ Y. Puttisong, I. A. Buyanova, A. J. Ptak, C. W. Tu, L. Geelhaar, H. Riechert, and W. M. Chen, Adv. Mater. 25, 738 (2013).

${ }^{15}$ Y. Puttisong, X. J. Wang, I. A. Buyanova, C. W. Tu, and W. M. Chen, J. Appl. Phys. 111, 07C303 (2012).

${ }^{16}$ Y. Puttisong, X. J. Wang, I. A. Buyanova, L. Geelhaar, H. Riechert, A. J. Ptak, C. W. Tu, and W. M. Chen, Nat. Commun. 4, 1751 (2013).

${ }^{17}$ Y. Araki, M. Yamaguchi, and F. Ishikawa, Nanotechnology 24, 065601 (2013).

${ }^{18}$ N. Ahn, Y. Araki, M. Kondow, M. Yamaguchi, and F. Ishikawa, Jpn. J. Appl. Phys., Part 1 53, 065001 (2014).

${ }^{19}$ I. A. Buyanova, W. M. Chen, G. Pozina, J. P. Bergman, H. P. Xin, and C. W. Tu, Appl. Phys. Lett. 75, 501 (1999).

${ }^{20}$ N. Q. Thinh, I. A. Buyanova, P. N. Hai, W. M. Chen, H. P. Xin, and C. W. Tu, Phys. Rev. B 63, 033203 (2001).

${ }^{21}$ X. J. Wang, Y. Puttisong, C. W. Tu, A. J. Ptak, V. K. Kalevich, A. Yu. Egorov, L. Geelhaar, H. Riechert, W. M. Chen, and I. A. Buyanova, Appl. Phys. Lett. 95, 241904 (2009).

${ }^{22}$ N. Q. Thinh, I. P. Vorona, I. A. Buyanova, W. M. Chen, S. Limpijumnong, S. B. Zhang, Y. G. Hong, H. P. Xin, C. W. Tu, A. Utsumi, Y. Furukawa, S. Moon, A. Wakahara, and H. Yonezu, Phys. Rev. B 71, 125209 (2005).

${ }^{23}$ A. M. Graham, P. Corfdir, M. Heiss, S. Conesa-Boj, E. Uccelli, A. Fontcuberta i Morral, and R. T. Phillips, Phys. Rev. B 87, 125304 (2013).

${ }^{24}$ P. Kusch, E. Grelich, C. Somaschini, E. Luna, M. Ramsteiner, L. Geelhaar, H. Riechert, and S. Reich, Phys. Rev. B 89, 045310 (2014).

${ }^{25} \mathrm{P}$. Corfdir, B. Hattem, E. Uccelli, A. Fontcuberta i Morral, and R. T. Phillips, Appl. Phys. Lett. 103, 133109 (2013).

${ }^{26}$ D. Spirkoska, J. Arbiol, A. Gustafsson, S. Conesa-Boj, F. Glas, I. Zardo, M. Heigoldt, M. H. Gass, A. L. Bleloch, S. Estrade, M. Kaniber, J. Rossler, F. Peiro, J. R. Morante, G. Abstreiter, L. Samuelson, and A. Fontcuberta i Morral, Phys. Rev. B 80, 245325 (2009). 
${ }^{27}$ L. Grenouillet, C. Bru-Chevallier, G. Guillot, P. Gilet, P. Duvaut, S. Vannuffel, A. Millon, and A. Chenevas-Paule, Appl. Phys. Lett. 76, 2241 (2000).

${ }^{28}$ I. A. Buyanova, W. M. Chen, and C. W. Tu, Solid State Electron. 47, 467 (2003).

${ }^{29}$ A. M. Mintairov, T. H. Kosel, J. L. Merz, P. A. Blagnov, A. S. Vlasov, V. M. Ustinov, and R. E. Cook, Phys. Rev. Lett. 87, 277401 (2001).

${ }^{30}$ I. Suemune, K. Uesugi, and W. Walukiewicz, Appl. Phys. Lett. 77, 3021 (2000).

${ }^{31}$ I. A. Buyanova, M. Izadifard, A. Kasic, H. Arwin, W. M. Chen, H. P. Xin, Y. G. Hong, and C. W. Tu, Phys. Rev. B 70, 085209 (2004).

${ }^{32}$ O. Demichel, M. Heiss, J. Bleuse, H. Mariette, and A. Fontcuberta i Morral, Appl. Phys. Lett. 97, 201907 (2010).

${ }^{33}$ S. Perera, M. A. Fickenscher, H. E. Jackson, L. M. Smith, J. M. YarrisonRice, H. J. Joyce, Q. Gao, H. H. Tan, C. Jagadish, X. Zhang, and J. Zou, Appl. Phys. Lett. 93, 053110 (2008).
${ }^{34}$ C.-C. Chang, C.-Y. Chi, M. Yao, N. Huang, C.-C. Chen, J. Theiss, A. W. Bushmaker, S. LaLumondiere, T.-W. Yeh, M. L. Povinelli, C. Zhou, P. D. Dapkus, and S. B. Cronin, Nano Lett. 12, 4484 (2012).

${ }^{35}$ D. J. Wolford, G. D. Gilliland, T. F. Kuech, J. F. Klem, H. P. Hjalmarson, J. A. Bradley, C. F. Tsang, and J. Martinsen, Appl. Phys. Lett. 64, 1416 (1994).

${ }^{36}$ H. H. Lee, R. J. Racicot, and S. H. Lee, Appl. Phys. Lett. 54, 724 (1989).

${ }^{37}$ A. Dobrovolsky, J. E. Stehr, S. L. Chen, Y. J. Kuang, S. Sukrittanon, C. W. Tu, W. M. Chen, and I. A. Buyanova, Appl. Phys. Lett. 101, 163106 (2012).

${ }^{38}$ G. W. p't Hooft, W. A. J. A. van der Poel, L. W. Molenkamp, and C. T. Foxon, Phys. Rev. B 35, 8281(R) (1987).

${ }^{39}$ J. E. Stehr, A. Dobrovolsky, S. Sukrittanon, Y. Kuang, C. W. Tu, W. M. Chen, and I. A. Buyanova, "Optimizing GaNP coaxial nanowires for efficient light emission by controlling formation of surface and interfacial defects," Nano Lett. (published online). 